\title{
Neuroendokrine Lungentumoren
}

\author{
Pulmonary Neuroendocrine Tumors
}

Autor

Institut

\section{Grohé}

Klinik für Pneumologie, Ev. Lungenklinik Berlin
Bibliografie

Dol http://dx.doi.org/ 10.1055/s-0034-1365409

Pneumologie 2014; 68: 455

(c) Georg Thieme Verlag KG

Stuttgart · New York

ISSN 0934-8387

Korrespondenzadresse

Prof. Dr. med. Christian Grohé

Klinik für Pneumologie,

Ev. Lungenklinik Berlin

Lindenberger Weg 27

13125 Berlin

Christian.Grohe@elk-berlin.de
Primär neuroendokrin differenzierte thorakale Tumore (PneuNET) der Lunge und des Mediastinums (2-4\% aller thorakalen Tumore, wenn die kleinzelligen Lungenkarzinome nicht berücksichtigt werden) zeichnen sich durch eine sehr unterschiedliche Morbidität und Mortalität aus. Während typische bronchopulmonale Karzinoide eine sehr gute Langzeitprognose aufweisen, sind großzellig neuroendokrine nicht-kleinzellige Lungenkarzinome als hochgradig maligne Tumoren mit sehr schlechter Überlebenswahrscheinlichkeit im metastasierten Stadium zu bewerten. Die vorliegende Übersichtsarbeit von Y. Sayeg et al. beschäftigt sich in ausführlicher Weise mit den klinischen Manifestationen, der Histopathologie und den zur Verfügung stehenden Therapieverfahren dieser schlecht verstandenen, jedoch faszinierend vielfältigen Entitäten.

Deutschlandweite Registerdaten für die Prävalenz, Therapieoptionen und Überlebensdaten der neuroendokrinen differenzierten Tumorentitäten der Thoraxorgane liegen nicht vor. Die aktuellen Bestrebungen einer personalisierten Medizin in der thorakalen Onkologie legen in Zukunft nahe, krankheitsentitätsbezogene Daten für diese heterogene Gruppe seltener Tumore in Deutschland zu erheben. Durch eine Initiative der Pneumologisch-Onkologischen Arbeitsgemeinschaft (POA) der Deutschen Krebsgesellschaft ergibt sich die Möglichkeit, die PneuNET-Patientendaten pseudonymisiert (nach entsprechender Einverständniserklärung und durch Ethikkommissionsvotum gestützt) prospektiv zu analysieren. In enger Kooperation mit dem exzellent etablierten Deutschen NET-Register für gastrointestinale NET der Deutschen Gesellschaft für Endokrinologie und in Zusammenarbeit mit dem Sekretär des NET-
Registers Herrn Dr. U.-F. Pape/Prof. Dr. B. Wiedenmann wurde ein Registersystem in Analogie zu dem NET-Register entwickelt. Das NET-Register stellt eine Plattform zur Registrierung und Erhebung von Daten neuroendokriner Tumorentitäten dar. Das NET-Register ist bereits sehr erfolgreich etabliert und biomathematisch validiert. Das durch die POA neu gegründete PneuNET-Register ermöglicht in Zukunft prospektiv Daten zu erheben und internetbasiert in der bereits etablierten NET-Plattform mit eigenem Modul einzugeben. Mit Ausnahme der kleinzelligen Lungenkarzinomdaten (SCLC) können Informationen zu neurokrin differenzierten thorakalen Tumoren (DIP-NECH, typisches Karzinoid, atypisches Karzinoid, großzellig neuroendokrin differenzierte, nicht-kleinzellige Lungenkarzinome (LCNEC) sowie mediastinale sich manifestierende neurokrin differenzierte Tumore wie zum Bespiel das Thymuskarzinoid bzw. das neurokrin endokrin differenzierte Thymuskarzinom) eingegeben werden. Durch die Zusammenarbeit der Kollegen aus der Thoraxklinik Heidelberg, Großhansdorf und Bad Berka (Prof. Dr. M. Thomas, Prof. Dr. H. Hoffmann, PD Dr. M. Reck, Dr. D. Heigener, Prof. Dr. D. Hörsch, Frau Dr. M. Serke) und der Ev. Lungenklinik Berlin-Buch wurde diese Initiative entwickelt. Die Plattform kann, in Ergänzung zu den bereits vor Ort bestehenden Tumordokumentationssystemen, über die Besonderheiten prognoserelevanter Parameter der neuroendokrin differenzierten thorakalen Tumoren spezifisch Auskunft geben. Die Analyse der personalisierten Therapieoptionen, die sich häufig multimodal gestalten, soll zukünftig zur Verbesserung der Prognose der Patienten beitragen. 